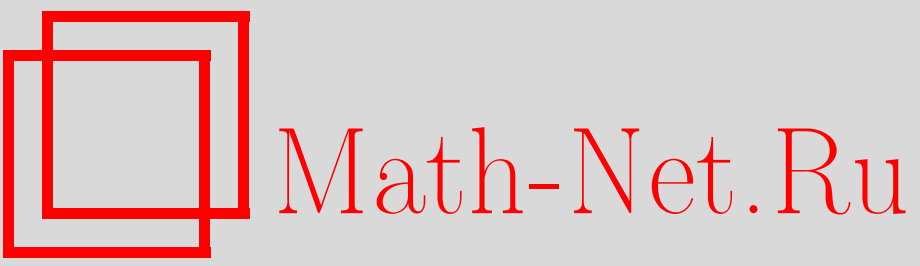

К. Л. Малышев, Функциональное интегрирование с "автоморфным" граничным условием и корреляторы третьих компонент спинов в $X X$-модели Гейзенберга, TMФ, 2003, том 136, номер 2, 285-298

DOI: https://doi.org/10.4213/tmf221

Использование Общероссийского математического портала Math-Net.Ru подразумевает, что вы прочитали и согласны с пользовательским соглашением

http://www.mathnet.ru/rus/agreement

Параметры загрузки:

IP : 3.85 .7 .115

26 апреля 2023 г., $14: 29: 53$ 


\section{ФУНКЦИОНАЛЬНОЕ ИНТЕГРИРОВАНИЕ С “АВТОМОРФНЫМ" ГРАНИЧНЫМ УСЛОВИЕМ И КОРРЕЛЯТОРЫ ТРЕТЬИХ КОМПОНЕНТ СПИНОВ В $X X$-МОДЕЛИ ГЕЙЗЕНБЕРГА}

Для производящей функции статических корреляторов третьих компонент спинов в $X X$-модели Гейзенберга получено новое представление в виде комбинации гауссовых функциональных интегралов по антикоммутирующим переменным. Специфика возникающих функциональных интегралов состоит в том, что часть переменных интегрирования зависит от мнимого времени "автоморфным" образом, т.е. умножается на определенное комплексное число при сдвиге мнимого времени на период. Остальные переменные подчиняются при этом обычным граничным условиям фермионного/бозонного типа. Результаты функционального интегрирования представлены в виде определителей матричных операторов. Окончательно производящая функция корреляторов и статистическая сумма модели вычисляются в подходе дзета-регуляризации. Корректность предложенного функционального определения подтверждается вычислением некоторых корреляционных функций третьих компонент спинов при ненулевой температуре.

Ключевые слова: функциональное интегрирование, $X X$-модель Гейзенберга, корреляторы, обобщенная дзета-функция.

Посвящается памяти А. Г. Изергина

\section{1. ВВЕДЕНИЕ}

Корреляционные функции квантовых моделей, решаемых с помошью анзаца Бете [1], связаны с определителями Фредгольма некоторых линейных интегральных операторов. Например, одно из таких детер.минантных представлений было получено в работах [2] для простейшего двух точечного одновременного коррелятора в одномерной модели "непроницаемых" бозонов, описываемых квантовым нелинейным уравнением Шредингера с бесконечным взаимодействием. Этот результат был обобшен на случай корреляторов

Результаты статьи были представлены на III Международном семинаре ПОМИ-Флоренция по квантовым группам и интегрируемым системам (Санкт-Петербург, 2-6 июля 2001 г.).

${ }^{*}$ Санкт-Петербургское отделение Математического института им. В. А. Стеклова РАН, СанктПетербург, Россия. E-mail: malyshev@pdmi.ras.ru 
с несовпадаюшими временнь́ми аргументами [3], а также для $X X$-модели Гейзенберга [4]. Детерминантные представления корреляционных функций позволяют получить классические нелинейные интегрируемые уравнения в частных производных, описывающие эти корреляторы [1], [5].

В свою очередь, имеется возможность вычислять средние квантовых операторов, пользуясь подходом функционального (континуального) интегрирования [6], [7]. В работах [8]-[10] подходы континуального, а также конечнократного интегрирования по антикоммутирующим переменным (по грассмановой алгебре [11]) используются при вычислении одновременных и разновременных температурных корреляторов различных компонент локальных спинов в $X X$ - и $X Y$-моделях Гейзенберга на периодической цепочке длины $M$.

Функциональные интегралы, полученные в работе [8] для производяшей функции корреляторов третьих компонент спинов в $X X$-модели, приводят к выражениям [12] в виде определителей конечномерных матриц размера $M \times M$. В работе [8] переменные интегрирования подчиняются граничным условиям по мнимому времени фермионного/бозонного типа. Уместно отметить, что $X X$-модель продолжает привлекать внимание [13]-[15] и детерминантные представления для корреляционных функций также активно изучаются [16].

В настояшей работе для $X X$-модели сформулирована другая версия представления производящей функции корреляторов третьих компонент спинов в виде комбинации функциональных интегралов. Развиваемый подход использует технический прием, который используется в работе [17], затрагивающей связь теорем об индексе с суперсимметричной квантовой механикой. А именно, для вычисления следов некоторых экспоненциальных операторов в [17] определяется функциональный интеграл по траекториям, преобразующимся "автоморфно" при сдвиге мнимого времени на период. Функциональные интегралы в данной работе также определены на множестве переменных, часть из которых зависит от мнимого времени “автоморфным" образом, т.е. умножается на определенное комплексное число при сдвиге мнимого времени на период, в то время как другая часть подчиняется стандартному условию фермионного/бозонного типа. При этом, в отличие от [17], возникает дополнительная необходимость восстановления инвариантности лагранжиана при с двиге мнимого времени на период. Для регуляризации возникающих определителей используется метод обобщенной дзета-функции. При этом оказывается, что и в подходе функционального интеграла можно достичь существенного упрощения и определенной наглядности. Некоторые подробности опущены, они могут быть найдены в работах [18].

В физическом контексте основные идеи дзета-регуляризации были сформулированы, например, в работах [19], [20]. Использование дзета-регуляризации для определения регуляризованных определителей операторов (ограниченных на ортогональное дополнение к своему ядру) в математической литературе связывается с работой [21]. Для физики дзета-регуляризация оказалась весьма полезным средством при вычислении, например, инстантонных детерминантов [22], [23], энергии Казимира на многообразиях [24], а также для вычисления аксиальной и конформной аномалий [25]. Для физических 
приложений дзета-регуляризация описана в работах [20], [26].

Работа организована следующим образом. Раздел 2 содержит основные обозначения и вводные замечания, касаюшиеся рассматриваемой $X X$-модели и производяшей функции корреляторов третьих компонент спинов. Вывод представлений для элементов производящей функции в виде гауссовых функциональных интегралов обсуждается в разделе 3 . В разделе 4 введена обобшенная дзета-функция в виде интеграла Меллина, и с ее помошью вычислены регуляризованные определители соответствуюших матричных операторов. В разделе 5 рассмотрено дифференцирование полученных представлений по параметру и вычислены некоторые конкретные корреляторы. Обсуждение в разделе 6 завершает работу.

\section{2. ОСНОВНЫЕ ОБОЗНАЧЕНИЯ}

Итак, рассмотрим, следуя [1], [4], [5], $X X$-модель Гейзенберга на периодической цепочке длины $M$ ( $M$ четно), взятую в представлении свободных фермионов [27]. Пусть $Q(m)$ есть оператор числа квазичастищ на первых $m$ узлах решетки $(m \leqslant M)$. Будем вычислять среднее оператора $e^{\alpha Q(m)}$ по основному состоянию модели (используемые обозначения стандартны, см. [4], [12])

$$
G(\alpha, m) \equiv\left\langle\Phi_{0}\left|e^{\alpha Q(m)}\right| \Phi_{0}\right\rangle=\frac{\operatorname{Tr}\left(e^{\alpha Q(m)} e^{-\beta H_{X X}}\right)}{\operatorname{Tr}\left(e^{-\beta H_{X X}}\right)}, \quad \alpha \in \mathbb{C},
$$

где $H_{X X}$ - гамильтониан $X X$-модели, $\beta$ - обратная температура и $\operatorname{Tr}$ означает след оператора. Используемый в предлагаемой работе гамильтониан $H_{X X}$ возникает в результате преобразования Йордана-Вигнера [27] от исходных матричных спиновых переменных на решетке к каноническим фермионным переменным с антикоммутационными соотношениями

$$
\left\{c_{k}, c_{n}\right\}=\left\{c_{k}^{\dagger}, c_{n}^{\dagger}\right\}=0, \quad\left\{c_{k}, c_{n}^{\dagger}\right\}=\delta_{k n} .
$$

В этих обозначениях $Q(m) \equiv \sum_{k=1}^{m} c_{k}^{\dagger} c_{k}$.

Можно проверить, что $H_{X X}$ перестановочен с оператором полного числа квазичастищ $\mathcal{N} \equiv Q(M)$, а оператор четности $(-1)^{\mathcal{N}}$ антикоммутирует с каноническими переменными и перестановочен с $H_{X X}$ как с оператором, билинейным по $c_{n}, c_{n}^{\dagger}$. Поэтому можно ввести два проектора $P^{ \pm}=\left(1 \pm(-1)^{\mathcal{N}}\right) / 2$ такие, что с их помощью $H_{X X}$ записывается в виде

$$
H_{X X}=H^{+} P^{+}+H^{-} P^{-}, \quad H^{ \pm}=-\frac{1}{2} \sum_{n=1}^{M}\left(c_{n}^{\dagger} c_{n+1}+c_{n+1}^{\dagger} c_{n}\right)+h \mathcal{N}-\frac{h M}{2},
$$

где $h>0$ - внешнее магнитное поле. Индекс \pm в (2) соответствует выбору граничных условий на цепочке в следуюшем виде:

$$
c_{M+1}=\mp c_{1}, \quad c_{M+1}^{\dagger}=\mp c_{1}^{\dagger}\left(\text { для } H^{ \pm}\right) .
$$


Учитывая соотношения (2), (3), искомое среднее будем вычислять по формуле [8]-[10]:

$$
G(\alpha, m)=\frac{1}{2 Z}\left(G_{\mathrm{F}}^{+} Z_{\mathrm{F}}^{+}+G_{\mathrm{F}}^{-} Z_{\mathrm{F}}^{-}+G_{\mathrm{B}}^{+} Z_{\mathrm{B}}^{+}-G_{\mathrm{B}}^{-} Z_{\mathrm{B}}^{-}\right),
$$

где

$$
\begin{aligned}
& G_{\mathrm{F}}^{ \pm} Z_{\mathrm{F}}^{ \pm} \equiv \operatorname{Tr}\left(e^{\alpha Q(m)} e^{-\beta H^{ \pm}}\right), \\
& G_{\mathrm{B}}^{ \pm} Z_{\mathrm{B}}^{ \pm} \equiv \operatorname{Tr}\left(e^{\alpha Q(m)}(-1)^{\mathcal{N}} e^{-\beta H^{ \pm}}\right)
\end{aligned}
$$

(индекс $X X$ опускаем). По аналогии с формулами (4а), (4б) статистическая сумма $Z=$ $\operatorname{Tr}\left(e^{-\beta H}\right)$ записывается в виде

$$
\begin{gathered}
Z=\frac{1}{2}\left(Z_{\mathrm{F}}^{+}+Z_{\mathrm{F}}^{-}+Z_{\mathrm{B}}^{+}-Z_{\mathrm{B}}^{-}\right) \\
Z_{\mathrm{F}}^{ \pm}=\operatorname{Tr}\left(e^{-\beta H^{ \pm}}\right), \quad Z_{\mathrm{B}}^{ \pm}=\operatorname{Tr}\left((-1)^{\mathcal{N}} e^{-\beta H^{ \pm}}\right) .
\end{gathered}
$$

В работе [17], затрагивающей связь теорем об индексе с суперсимметричной квантовой механикой, содержится следуюшее наблюдение. Пусть $a, a^{\dagger}-$ фермиевские канонические операторы. Рассмотрим $\mathcal{U}(1)$-оператор $Q_{\vartheta} \equiv e^{i \vartheta a^{\dagger} a}$, действуюший на $a, a^{\dagger}$ следуюшим образом:

$$
Q_{\vartheta} a Q_{\vartheta}^{\dagger}=e^{-i \vartheta} a, \quad Q_{\vartheta} a^{\dagger} Q_{\vartheta}^{\dagger}=e^{i \vartheta} a^{\dagger} .
$$

Оказывается, что при вычислении $\operatorname{Tr}\left(Q_{\vartheta} e^{-\beta H}\right)$ ( $H$ - соответствующий гамильтониан) можно естественно прийти к функциональному интегралу по переменной, подчиненной условию $\xi(\tau)=-e^{i \vartheta} \xi(\tau+\beta)$, означаюшему, что при сдвиге $\tau$ на период $\beta$ переменная функционального интегрирования преобразуется по представлению $\mathcal{U}(1)$, т.е. "автоморфно" [28]. Заметим, что "автоморфная" зависимость от $\tau$ возникла также в работе [29] при исследовании некоторых квантово-статистических спиновых моделей в подходе функционального интегрирования.

Нетрудно заметить, что оператор $e^{\alpha Q(m)}$ обладает аналогичным свойством:

$$
e^{\alpha Q(m)} c_{n} e^{-\alpha Q(m)}= \begin{cases}e^{-\alpha} c_{n}, & 1 \leqslant n \leqslant m, \\ c_{n} & m<n \leqslant M,\end{cases}
$$

и при вычислении (1) можно использовать идею [17]. "Автоморфное” условие возникает в работах [17] и [29] на всех узлах рассматриваемых там моделей. Можно догадаться, что специфика при выгислении (1) окажется связанной с условием $m \leqslant M$.

В заключение раздела введем необходимые нам когерентные состояния, отвечаюшие фермионным операторам $c_{n}, c_{n}^{\dagger}$, для которых имеется фоковский вакуум $|0\rangle$ :

$$
c_{n}|0\rangle=\langle 0| c_{n}^{\dagger}=0 \quad \forall n, \quad\langle 0 \mid 0\rangle=1 .
$$

А именно, определим состояния

$$
\begin{gathered}
|x(a)\rangle=\exp \left\{\sum_{k=1}^{M} c_{k}^{\dagger} x_{k}(a)\right\}|0\rangle \equiv e^{c^{\dagger} x(a)}|0\rangle \\
\left\langle x^{*}(a)\right|=\langle 0| \exp \left\{\sum_{k=1}^{M} x_{k}^{*}(a) c_{k}\right\} \equiv\langle 0| e^{x^{*}(a) c}
\end{gathered}
$$


где $a$ - дискретный индекс, принимающий значения от 1 до $N$, и использованы сокрашенные обозначения $\sum_{k=1}^{M} c_{k}^{\dagger} x_{k} \equiv c^{\dagger} x, \prod_{k=1}^{M} d x_{k} \equiv d x$ и т.д. Фактически определены $N$ независимых когерентных состояний, зависяших от комплекснозначных грассмановых параметров $x_{k}^{*}(a), x_{k}(a)$. Векторы (7) оказываются собственными для оператоpoв $c_{k}, c_{k}^{\dagger}$ :

$$
\begin{gathered}
c_{k}|x(a)\rangle=x_{k}(a)|x(a)\rangle, \quad\left\langle x^{*}(a)\right| c_{k}^{\dagger}=\left\langle x^{*}(a)\right| x_{k}^{*}(a), \\
\left\langle x^{*}(a) \mid x(a)\right\rangle=e^{x^{*}(a) x(a)}
\end{gathered}
$$

\section{3. ФУНКЦИОНАЛЬНЫЙ ИНТЕГРАЛ}

Перейдем к выводу представлений для $(4 \sigma),(5)$ в виде функциональных интегралов. Рассмотрим для определенности

$$
G_{\mathrm{F}}^{ \pm} Z_{\mathrm{F}}^{ \pm}=\int d z d z^{*} e^{z^{*} z}\left\langle z^{*}\left|e^{\alpha Q(m)} e^{-\beta H^{ \pm}}\right| z\right\rangle
$$

где след оператора записан как интеграл по антикоммутирующим переменным [11] и векторы $\left\langle z^{*}|| z,\right\rangle$ определены аналогично (7). Для перехода к функциональному интегралу разделим отрезок $[0, \beta]$ на $N$ частей длины $\beta / N$ и представим $e^{-\beta H^{ \pm}}$в виде произведения $N$ одинаковых сомножителей. Вставляя между этими сомножителями $N$ разложений единицы, преобразуем (8) к виду

$$
\begin{aligned}
G_{\mathrm{F}}^{ \pm} Z_{\mathrm{F}}^{ \pm}= & \int d z d z^{*} \prod_{a=1}^{N} d x^{*}(a) d x(a) \exp \left(z^{*} z-\sum_{a=1}^{N} x^{*}(a) x(a)\right) \times \\
& \times\left\langle z^{*}\left|e^{\alpha Q(m)}\right| x(1)\right\rangle\left\langle x^{*}(1)\left|e^{-\frac{\beta}{N} H^{ \pm}}\right| x(2)\right\rangle \ldots\left\langle x^{*}(N)\left|e^{-\frac{\beta}{N} H^{ \pm}}\right| z\right\rangle,
\end{aligned}
$$

где $|x(a)\rangle$ и $\left\langle x^{*}(a)\right|$ определены в (7).

Используя свойства когерентных состояний, находим матричные элементы

$$
\begin{aligned}
&\left\langle z^{*}\left|e^{\alpha Q(m)}\right| x(1)\right\rangle= \exp \left(e^{\alpha} \sum_{k=1}^{m} z_{k}^{*} x_{k}(1)+\sum_{k=m+1}^{M} z_{k}^{*} x_{k}(1)\right) \\
&\left\langle x^{*}(a)\left|e^{-\frac{\beta}{N} H^{ \pm}}\right| x(a+1)\right\rangle \simeq \exp \left(x^{*}(a) x(a+1)-\frac{\beta}{N} H^{ \pm}\left(x^{*}(a), x(a+1)\right)\right), \quad N \gg 1, \\
& H^{ \pm}\left(x^{*}(a), x(a+1)\right) \equiv-\frac{1}{2} \sum_{k=1}^{M}\left(x_{k}^{*}(a) x_{k+1}(a+1)+x_{k+1}^{*}(a) x_{k}(a+1)\right)+ \\
&+h \sum_{k=1}^{M} x_{k}^{*}(a) x_{k}(a+1)-\frac{h M}{2}
\end{aligned}
$$


и, подставляя их в (9), получаем

$$
\begin{aligned}
G_{\mathrm{F}}^{ \pm} Z_{\mathrm{F}}^{ \pm}= & \int d z d z^{*} \prod_{a=1}^{N} d x^{*}(a) d x(a) \exp \left\{\sum_{k=1}^{m} z_{k}^{*}\left(z_{k}+e^{\alpha} x_{k}(1)\right)+\right. \\
& +\sum_{k=m+1}^{M} z_{k}^{*}\left(z_{k}+x_{k}(1)\right)+x^{*}(1)(x(2)-x(1))+\cdots+x^{*}(N)(z-x(N))- \\
& \left.-\frac{\beta}{N}\left(H^{ \pm}\left(x^{*}(1), x(2)\right)+\cdots+H^{ \pm}\left(x^{*}(N), z\right)\right)\right\}
\end{aligned}
$$

Введем обозначения $x_{k}(N+1) \equiv z_{k}(\forall k), x_{k}^{*}(0) \equiv e^{\alpha} z_{k}^{*}$ (для $\left.1 \leqslant k \leqslant m\right)$ и $x_{k}^{*}(0) \equiv z_{k}^{*}$ (для $m<k \leqslant M$ ). Далее, наложим условия

$$
x_{k}(0)= \begin{cases}-e^{-\alpha} x_{k}(N+1), & 1 \leqslant k \leqslant m, \\ -x_{k}(N+1), & m<k \leqslant M,\end{cases}
$$

и сделаем переход $N \rightarrow \infty$. В результате дискретный индекс $a$, меняюшийся от 1 до $N$, превратится в непрерывный аргумент $\tau \in[0, \beta]$ ("мнимое" время [7], [11]). При этом правая часть (10) преврашается в интеграл

$$
\int \prod_{\tau \in[0, \beta]} d x^{*}(\tau) d x(\tau) \exp \left(\int_{0}^{\beta} \mathcal{L}(\tau) d \tau\right),
$$

где лагранжиан $\mathcal{L}(\tau)$ имеет вид

$$
\mathcal{L}(\tau)=x^{*}(\tau) \frac{d x}{d \tau}-H^{ \pm}\left(x^{*}(\tau), x(\tau)\right)
$$

и функциональные переменные $x(\tau)$ подчинены “автоморфному" граничному условию

$$
x_{k}(\tau)= \begin{cases}-e^{-\alpha} x_{k}(\tau+\beta), & 1 \leqslant k \leqslant m, \\ -x_{k}(\tau+\beta), & m<k \leqslant M .\end{cases}
$$

Поля $x_{k}^{*}(\tau)$ - независимые переменные интегрирования. Оказывается удобным подчинить $x_{k}^{*}(\tau)$ требованию, аналогичному (11в), но с фактором $e^{\alpha}$ вместо $e^{-\alpha}$.

Проделанный вывод представления (11a)-(11в) наивно следует [17] и не учитывает спешифику квадратичных гамильтонианов $H^{ \pm}(2)$. Можно считать, что как традиционные условия (анти)периодичности $x_{k}(\tau)= \pm x_{k}(\tau+\beta), k \in\{1, \ldots, M\}$, так и условия (11в) задают некоторое представление относительно сдвигов $\tau$ на период $\beta$. Действие $\int_{0}^{\beta} \mathcal{L}(\tau) d \tau$, стояшее в показателе экспоненты интеграла (11a), является корректным объектом при условии инвариантности $\mathcal{L}(\tau)$ относительно сдвига $\tau$. В случае стандартных условий по $\tau$ такая инвариантность имеет место, если $\mathcal{L}(\tau)$ содержит только четные степени полей. 
Используем (11в) для вычисления прирашения $\delta \mathcal{L}(\tau)$ :

$$
\begin{aligned}
\delta \mathcal{L}(\tau)= & \mathcal{L}(\tau+\beta)-\mathcal{L}(\tau)= \\
= & \frac{1}{2}\left[\left(e^{\alpha}-1\right)\left(x_{m+1}^{*}(\tau) x_{m}(\tau)+x_{M}^{*}(\tau) x_{M+1}(\tau)\right)+\right. \\
& \left.+\left(e^{-\alpha}-1\right)\left(x_{m}^{*}(\tau) x_{m+1}(\tau)+x_{M+1}^{*}(\tau) x_{M}(\tau)\right)\right] .
\end{aligned}
$$

Причина возникновения приращения $\delta \mathcal{L}(\tau)$ проста: циклические квадратичные формы $H^{ \pm}$инвариантны при замене

$$
x_{k} \rightarrow \pm e^{\alpha} x_{k}, \quad x_{k}^{*} \rightarrow \pm e^{-\alpha} x_{k}^{*}
$$

если $k \in\{1, \ldots, M\}$ (однородное “калибровочное" преобразование), и неинвариантны, если замена (12) затрагивает только часть узлов (неоднородное преобразование). Условие (11в) задает на переменных интегрирования неоднородное представление группы сдвигов $\tau \rightarrow \tau+\beta$, и для $\mathcal{L}(\tau)$ (11б) инвариантность оказывается нарушенной. Эту симметрию легко восстановить в предельной формуле $(11 \mathrm{a})$, заменив $H^{ \pm}\left(x^{*}(\tau), x(\tau)\right)$ на форму следуюшего вида:

$$
\begin{aligned}
\widetilde{H}^{ \pm}(\tau)= & -\frac{1}{2} \sum_{k=1}^{M}\left(x_{k+1}^{*}(\tau) x_{k}(\tau)+x_{k}^{*}(\tau) x_{k+1}(\tau)\right)+ \\
& +h \sum_{k=1}^{M} x_{k}^{*}(\tau) x_{k}(\tau)-\frac{1}{2}\left[x_{m+1}^{*}(\tau) x_{m}(\tau) e^{-\frac{\alpha}{\beta} \tau}+x_{m}^{*}(\tau) x_{m+1}(\tau) e^{\frac{\alpha}{\beta} \tau}+\right. \\
& \left.+x_{M+1}^{*}(\tau) x_{M}(\tau) e^{\frac{\alpha}{\beta} \tau}+x_{M}^{*}(\tau) x_{M+1}(\tau) e^{-\frac{\alpha}{\beta} \tau}\right]-\frac{h M}{2}
\end{aligned}
$$

где символ $\sum^{\prime}$ означает, что при суммировании пропушены индексы $k=m, M$. Лагранжиан

$$
\widetilde{\mathcal{L}}(\tau)=x^{*}(\tau) \frac{d x}{d \tau}-\widetilde{H}^{ \pm}(\tau)
$$

инвариантен относительно сдвига $\tau \rightarrow \tau+\beta$, мера в интеграле (11a) также инвариантна, и окончательно получаем

$$
G_{\mathrm{F}}^{ \pm} Z_{\mathrm{F}}^{ \pm}=\int \prod_{\tau \in[0, \beta]} d x^{*}(\tau) d x(\tau) \exp \left(\int_{0}^{\beta} \widetilde{\mathcal{L}}(\tau) d \tau\right)
$$

Утверждение данной работы состоит в том, что представление (13), дополненное условием (11в), является корректным соотношением, альтернативным представлению, полученному в работе [8]. Последуюшие вычисления демонстрируют это.

Перейдем в (13) к импульсному представлению:

$$
x_{k}(\tau)= \begin{cases}(\beta M)^{-1 / 2} \sum_{p} e^{i\left(\omega \tau-i \frac{\alpha}{\beta} \tau+q k\right)} x_{p}, & 1 \leqslant k \leqslant m, \\ (\beta M)^{-1 / 2} \sum_{p} e^{i(\omega \tau+q k)} x_{p}, & m<k \leqslant M,\end{cases}
$$


где индекс суммирования $p \equiv(\omega, q)$ - формальный 2-импульс: суммирование идет по $\omega=\pi T(2 n+1), n \in \mathbb{Z}$, и по квазиимпульсам $q \in X^{ \pm}$. Условие (11в) приводит к тому, что в фурье-разложениях на первых $m$ узлах фермиевские частоты оказываются сдвинутыми на чисто мнимое число. Однако индексом суммирования в (14a) можно, конечно, считать $\omega$. Два множества $X^{ \pm}$,

$$
\begin{aligned}
& X^{+}=\left\{q=-\pi+\frac{\pi(2 l-1)}{M} \mid l=1, \ldots, M\right\}, \\
& X^{-}=\left\{q=-\pi+\frac{2 \pi l}{M} \mid l=1, \ldots, M\right\},
\end{aligned}
$$

возникают благодаря двум граничным условиям (3).

Подставим разложения $(14 \mathrm{a})$ в $(13)$, перенормируем $x_{p} e^{i(m+1) q / 2} \rightarrow x_{p}$ и получим

$$
\begin{gathered}
G_{\mathrm{F}}^{ \pm} Z_{\mathrm{F}}^{ \pm}=\int \prod_{p} d x_{p}^{*} d x_{p} e^{S^{ \pm}(\alpha)}, \\
S^{ \pm}(\alpha)=\sum_{p}\left(i \omega-\varepsilon_{q}\right) x_{p}^{*} x_{p}+\frac{\alpha}{\beta} \sum_{\omega, q, q^{\prime}} Q_{q q^{\prime}} x_{\omega q}^{*} x_{\omega q^{\prime}}+\frac{M h \beta}{2},
\end{gathered}
$$

где $\varepsilon_{q}=h-\cos q-$ зонная энергия квазичастиц, $p$ пробегает все допустимые значения,

$$
Q_{q q^{\prime}}=\frac{1}{M} \frac{\sin \left(m\left(q-q^{\prime}\right) / 2\right)}{\sin \left(\left(q-q^{\prime}\right) / 2\right)}
$$

и $q, q^{\prime} \in X^{+}$либо $X^{-}$. Таким образом, “автоморфное” условие (11в) на первых $m$ узлах ответственно за возникновение недиагонального члена в $S^{ \pm}(\alpha)$. При $m=M$ величина $Q_{q q^{\prime}}$ становится символом Кронекера и $S^{ \pm}(\alpha)$ превращается в свободное фермионное действие со сдвинутым химическим потенциалом [29].

Воспользуемся известным результатом для конечнократных интегралов [7], [11] и получим следуюшие формальные выражения:

$$
\begin{aligned}
& G_{\mathrm{F}}^{ \pm} Z_{\mathrm{F}}^{ \pm}=e^{M h \beta / 2} \operatorname{Det}\left[\left(-i \omega_{\mathrm{F}}+\varepsilon_{q}\right) \delta_{p p^{\prime}}-\frac{\alpha}{\beta} \delta_{\omega \omega^{\prime}} Q_{q q^{\prime}}\right] \\
& G_{\mathrm{B}}^{ \pm} Z_{\mathrm{B}}^{ \pm}=e^{M h \beta / 2} \operatorname{Det}\left[\left(-i \omega_{\mathrm{B}}+\varepsilon_{q}\right) \delta_{p p^{\prime}}-\frac{\alpha}{\beta} \delta_{\omega \omega^{\prime}} Q_{q q^{\prime}}\right]
\end{aligned}
$$

$$
\begin{aligned}
& Z_{\mathrm{F}}^{ \pm}=e^{M h \beta / 2} \operatorname{Det}\left[\left(i \omega_{\mathrm{F}}-\varepsilon_{q}\right) \delta_{p p^{\prime}}\right] \\
& Z_{\mathrm{B}}^{ \pm}=e^{M h \beta / 2} \operatorname{Det}\left[\left(i \omega_{\mathrm{B}}-\varepsilon_{q}\right) \delta_{p p^{\prime}}\right]
\end{aligned}
$$

где $\omega_{\mathrm{F}}$ и $\omega_{\mathrm{B}}-$ фермиевские и бозевские частоты. Модификация вычислений для $G_{\mathrm{B}}^{ \pm} Z_{\mathrm{B}}^{ \pm}$ (4б) проводится совершенно аналогично, но присутствие $(-1)^{\mathcal{N}}$ под знаком следа приводит к замене двух знаков минус (после равенства) в условии (11в) на знаки плюс. В 
результате параметром суммирования оказывается не фермиевская частота $\omega_{\mathrm{F}}$, а бозевская частота $\omega_{\mathrm{B}}$ (т.е. $\omega=2 \pi T n, n \in \mathbb{Z}$ ) и ответы для $G_{\mathrm{B}}^{ \pm} Z_{\mathrm{B}}^{ \pm}$и $Z_{\mathrm{B}}^{ \pm}$принимают вид (16б) и (17б), соответственно. Здесь и ниже символ Det используется для обозначения определителя бесконечномерных матриц, det - для конечномерных. Подстановка (16a), (16б) в (4a) приводит к ответу для $G(\alpha, m)$, а статсумма $Z$ вычисляется с помощью (5) и (17a), (17б). Матричные операторы, возникающие в (16a), (16б) и (17a), (17б), удобно обозначать соответственно $A(\alpha) \equiv A_{\alpha}$ и $A$.

\section{4. ДЗЕТА-РЕГУЛЯРИЗАЦИЯ}

Для придания смысла определителям (16), (17) в данной работе предлагается использовать дзета-регуляризацию [20], [21], [26]. Обычно обобшенная дзета-функция связана с некоторым эллиптическим оператором. А именно, пусть $\mathcal{A}$ - неотрицательный эллиптический оператор порядка $p>0$ на компактном $d$-мерном гладком многообразии. Пусть его собственные значения $\lambda_{n}$ нумеруются мультииндексом $n$. Обобшенной дзета-функцией оператора $\mathcal{A}$ назовем ряд

$$
\zeta(s \mid \mathcal{A})=\sum_{\lambda_{n} \neq 0}\left(\lambda_{n}\right)^{-s}
$$

сходящийся при $\operatorname{Re} s>d / p$. Этот ряд определяет $\zeta(s \mid \mathcal{A})$ как мероморфную функцию переменной $s \in \mathbb{C}$, обладающую аналитическим продолжением в точку $s=0$. Соотношение (18) позволяет записать регуляризованный определитель $\mathcal{A}$ в виде

$$
\ln \operatorname{Det} \mathcal{A}=-\zeta^{\prime}(0 \mid \mathcal{A}) \text {. }
$$

Отправляясь от $(18)$, можно получить представление $\zeta(s \mid \mathcal{A})$ в виде интеграла Меллина:

$$
\zeta(s \mid \mathcal{A})=\frac{1}{\Gamma(s)} \int_{0}^{\infty} t^{s-1}\left[\operatorname{Tr}\left(e^{-\mathcal{A} t}\right)-\operatorname{dim}(\operatorname{ker} \mathcal{A})\right] d t
$$

Интеграл (20) определен при $\operatorname{Re} s>d / p$, при остальных значениях $\operatorname{Re} s$ требуется его аналитическое продолжение. Формулу (20) можно связать [26] с определением Det $\mathcal{A}$ с помощью регуляризации по собственному времени [30]. Используем (20) для придания смысла определителям (16), (17).

Рассмотрим, например, $(16 \mathrm{a})$ и вычислим $G_{\mathrm{F}}^{ \pm}$. Запишем формальный интеграл

$$
\frac{1}{\Gamma(s)} \int_{0}^{\infty} t^{s-1} \operatorname{Tr}\left[e^{\left(i \omega_{\mathrm{F}}-\hat{\varepsilon}+\frac{\alpha}{\beta} \widehat{Q}\right) t}\right] d t
$$

где $\hat{\varepsilon}$ и $\widehat{Q}$ означают матрицы в импульсном пространстве соответственно $\operatorname{diag}\left\{\varepsilon_{q}\right\}$ и $Q_{p q}$ (15б), а индексы $p, q$ независимо пробегают множества $X^{+}$или $X^{-}$. Сходимость интеграла (21) на верхнем пределе обеспечена при $\varepsilon_{q}>0$ (т.е. при $h>h_{\mathrm{c}}=1, h_{\mathrm{c}}$ - критическое магнитное поле [4]); на нижнем пределе требуется регуляризация.

Воспользуемся асимптотическим соотношением

$$
\operatorname{Tr}\left[e^{\left(i \omega_{\mathrm{F}}-\hat{\varepsilon}+\frac{\alpha}{\beta} \hat{Q}\right) t}\right] \longrightarrow \phi_{0}, \quad t \rightarrow 0
$$


где $\phi_{0} \equiv \phi_{0}\left(A_{\alpha}\right)$ - бесконечная постоянная, равная $\operatorname{Tr}\left(\delta_{p p^{\prime}}\right) \equiv \sum_{\omega_{\mathrm{F}}} \operatorname{tr}(\widehat{\delta})(\widehat{\delta}$ - единичная матрица размера $M \times M)$, и определим функцию $\rho(t)$ :

$$
\rho(t) \equiv \operatorname{Tr}\left[e^{\left(i \omega_{\mathrm{F}}-\hat{\varepsilon}+\frac{\alpha}{\beta} \widehat{Q}\right) t}\right]-\phi_{0}, \quad 0 \leqslant t<1 .
$$

Разобьем интеграл (21) на две части и запишем его с помошью (22) следуюшим образом:

$$
\frac{1}{\Gamma(s)} \int_{1}^{\infty} t^{s-1} \operatorname{Tr}\left[e^{\left(i \omega_{\mathrm{F}}-\hat{\varepsilon}+\frac{\alpha}{\beta} \widehat{Q}\right) t}\right] d t+\frac{1}{\Gamma(s)} \int_{0}^{1} t^{s-1} \rho(t) d t+\frac{\phi_{0}}{s \Gamma(s)} .
$$

Функция $\rho(t)$ есть формальньй ряд по степеням $t^{n}, n \geqslant 1$. Кроме того,

$$
\frac{1}{s \Gamma(s)} \simeq 1+\gamma s+o(s), \quad \gamma=-\psi(1)
$$

где $\psi(z)=(d / d z) \ln \Gamma(z)$. Следовательно, выражение (23), аналитичное при $s \rightarrow 0$, определяет аналитическое продолжение (21) при всех $\operatorname{Re} s \geqslant 0$, и именно его можно считать определением соответствуюшей обобщенной дзета-функции матричного оператора $A_{\alpha}(16 \mathrm{a})$ в правой полуплоскости $\mathbb{C} \ni s$.

Рассмотрим теперь вопрос о постоянной $\phi_{0}$ и о коэффициентах, характеризуюших $\rho(t)$. В случае дифференциальных операторов на многообразиях эти числа (коэффициенты Сили) оказываются ненулевыми [25], [26]. В нашем случае эти коэффициенты бесконечны, однако им могут быть приписаны [18] конечные (фактически нулевые) значения, если использовать известные аналитические продолжения [31] для дзета-функции Римана $\zeta(s)$ и обобшенной дзета-функции $\zeta(s, \alpha)$.

Воспользуемся формулой (24) и перейдем от (23) к соотношению

$$
-\lim _{s \rightarrow 0} \frac{d}{d s} \zeta_{\mathrm{F}}^{ \pm}\left(s \mid A_{\alpha}\right)=-\int_{1}^{\infty} \operatorname{tr}\left[e^{\left(-\hat{\varepsilon}+\frac{\alpha}{\beta} \widehat{Q}\right) t}\right]\left(\sum_{\omega_{\mathrm{F}}} e^{i \omega_{\mathrm{F}} t}\right) \frac{d t}{t}-\int_{0}^{1} \rho(t) \frac{d t}{t}-\gamma \phi_{0},
$$

где можно использовать формулу Пуассона для суммирования по $\omega_{F}$. Тогда правая часть (25) принимает вид

$$
-\operatorname{tr} \sum_{k=1}^{\infty} \frac{(-1)^{k}}{k}\left(e^{-\beta \hat{\varepsilon}+\alpha \widehat{Q}}\right)^{k}-\int_{0}^{1} \rho(t) \frac{d t}{t}-\gamma \phi_{0}=\ln \operatorname{det}\left(1+e^{-\beta \hat{\varepsilon}+\alpha \widehat{Q}}\right)-\int_{0}^{1} \rho(t) \frac{d t}{t}-\gamma \phi_{0} .
$$

Учтем также, что $\widehat{Q}^{2}=\widehat{Q}$, и поэтому $e^{\alpha \widehat{Q}}-1=\left(e^{\alpha}-1\right) \widehat{Q}$. Следовательно,

$$
G_{\mathrm{F}}^{ \pm}=\frac{\operatorname{Det}\left[\left(i \omega_{\mathrm{F}}-\varepsilon_{q}\right) \delta_{p p^{\prime}}+\frac{\alpha}{\beta} \delta_{\omega \omega^{\prime}} Q_{q q^{\prime}}\right]}{\operatorname{Det}\left[\left(i \omega_{\mathrm{F}}-\varepsilon_{q}\right) \delta_{p p^{\prime}}\right]}=\operatorname{det}\left[1+\left(e^{\alpha}-1\right) \widehat{Q}\left(1+e^{\beta \hat{\varepsilon}}\right)^{-1}\right]
$$

Действуя аналогично, для $G_{\mathrm{B}}^{ \pm}(16 \sigma)$ получаем

$$
G_{\mathrm{B}}^{ \pm}=\operatorname{det}\left[1+\left(e^{\alpha}-1\right) \widehat{Q}\left(1-e^{\beta \hat{\varepsilon}}\right)^{-1}\right] .
$$


Уже представление (23) (с учетом замечания о конечности $\rho(t)$ и $\phi_{0}$ ) позволяет обсуждать регуляризованный $\operatorname{Det} A_{\alpha}$. Однако для $G_{\mathrm{F}}^{ \pm}, G_{\mathrm{B}}^{ \pm}$, т.е. для интересуюших нас отношений определителей, дополнительная ренормировка $\rho(t)$ и $\phi_{0}$ не имеет значения. Выполнение в (26) (без двух последних слагаемых) предела $\alpha \rightarrow 0$ приводит к правильному ответу [9], [12] для $Z_{\mathrm{F}}^{ \pm}, Z_{\mathrm{B}}^{ \pm}$:

$$
\begin{aligned}
& Z_{\mathrm{F}}^{ \pm}=e^{M h \beta / 2} \prod_{q \in X^{ \pm}}\left(1+e^{-\beta \varepsilon_{q}}\right)=\prod_{q \in X^{ \pm}} 2 \operatorname{ch} \frac{\beta \varepsilon_{q}}{2}, \\
& Z_{\mathrm{B}}^{ \pm}=e^{M h \beta / 2} \prod_{q \in X^{ \pm}}\left(1-e^{-\beta \varepsilon_{q}}\right)=\prod_{q \in X^{ \pm}} 2 \operatorname{sh} \frac{\beta \varepsilon_{q}}{2},
\end{aligned}
$$

что подтверждает корректность соотношений $(23),(25),(26)$.

Мы ограничились случаем $h>1$ при выводе (27), (28). В случае $0<h<1$ величина $\varepsilon_{q}$ не является строго положительной, и может возникнуть вопрос о сходимости интеграла (21) на верхнем пределе. Можно показать, что в этом случае надо вычислять регуляризованное значение $\operatorname{Det}^{1 / 2}\left(A_{\alpha} A_{\alpha}^{*}\right)$, где $A_{\alpha}^{*}$ - комплексно-сопряженный оператор, и окончательный результат совпадет с $(27),(28)$. Случай $0<h<1$ может быть опушен для краткости при обсуж дении возможности возникновения "автоморфных" граничных условий (11в) при вычислении (1).

\section{5. ДИФФЕРЕНЦИРОВАНИЕ ОПРЕДЕЛИТЕЛЕЙ}

При вычислении корреляторов третьих компонент спинов (оператор третьей компоненты спина на узле $\sigma_{z}^{(m)}$, как и другие локальные спиновые операторы, определен, например, в [4]) приходится дифференцировать (1) по $\alpha$ в точке $\alpha=0$, т.е. вычислять средние вида

$$
\left\langle Q^{n}(m)\right\rangle=\lim _{\alpha \rightarrow 0} \frac{d^{n}}{d \alpha^{n}} G(\alpha, m)
$$

(см. [1], [4]). Фактически, имея в виду предложенное представление (23), достаточно научиться вычислять только первую производную. Остальные дифференцирования будут проходить как обычные дифференцирования конечномерных матриц.

Рассматриваемые операторы $A(\alpha)$ линейны по $\alpha: A(\alpha) \equiv A_{1}+\alpha A_{2}$. Вычислим в качестве примера первую производную по $\alpha$ от определителя Det $A(\alpha)(16 \mathrm{a})$, используя формальный интеграл (21):

$$
\frac{(d / d \alpha) \operatorname{Det} A(\alpha)}{\operatorname{Det} A(\alpha)}=-\frac{d}{d \alpha}\left(\int_{0}^{\infty} \operatorname{Tr}\left(e^{A(\alpha) t}\right) \frac{d t}{t}\right)
$$

(для дифференцирования по параметру регуляризация при $t \rightarrow+0$ несушественна). В духе леммы Рэя-Зингера-Шварца [26], [32] используем в (30) следуюшее равенство:

$$
\frac{d}{d \alpha}\left(\operatorname{Tr}\left(e^{A(\alpha) t}\right)\right)=t \frac{d}{d t} \operatorname{Tr}\left(B(\alpha) e^{A(\alpha) t}\right), \quad B(\alpha) \equiv A_{2} A^{-1}(\alpha) .
$$


Тогда интеграл по $t$ можно вычислить, получаем

$$
\frac{(d / d \alpha) \operatorname{Det} A_{\alpha}}{\operatorname{Det} A_{\alpha}}=\operatorname{Tr}\left(\frac{\widehat{Q}}{\beta}\left(i \omega_{\mathrm{F}}-\hat{\varepsilon}+\frac{\alpha}{\beta} \widehat{Q}\right)^{-1}\right)=\operatorname{tr}\left(\widehat{Q}\left(1+e^{\beta \hat{\varepsilon}-\alpha \widehat{Q}}\right)^{-1}\right),
$$

где при суммировании по частотам $\omega_{\mathrm{F}}$ использована формула Коши для матриц [33]. С учетом (31) можно вычислить все нужные производные:

$$
\begin{aligned}
\lim _{\alpha \rightarrow 0} \frac{(d / d \alpha) \operatorname{Det} A_{\alpha}}{\operatorname{Det} A_{\alpha}}= & \operatorname{tr}\left(\widehat{Q}\left(1+e^{\beta \hat{\varepsilon}}\right)^{-1}\right) \\
\lim _{\alpha \rightarrow 0} \frac{\left(d^{2} / d \alpha^{2}\right) \operatorname{Det} A_{\alpha}}{\operatorname{Det} A_{\alpha}}= & \operatorname{tr}\left(\widehat{Q}\left(1+e^{\beta \hat{\varepsilon}}\right)^{-1}\right)+\operatorname{tr}^{2}\left(\widehat{Q}\left(1+e^{\beta \hat{\varepsilon}}\right)^{-1}\right)- \\
& -\operatorname{tr}\left(\widehat{Q}\left(1+e^{\beta \hat{\varepsilon}}\right)^{-1} \widehat{Q}\left(1+e^{\beta \hat{\varepsilon}}\right)^{-1}\right)
\end{aligned}
$$

и т.д.

В завершение раздела используем полученные формулы для вычисления корреляторов $\left\langle\sigma_{z}^{(m)}\right\rangle$ и $\left\langle\sigma_{z}^{(m+1)} \sigma_{z}^{(1)}\right\rangle$ в термодинамическом пределе. В этом случае бозонные вклады $G_{\mathrm{B}}^{+} Z_{\mathrm{B}}^{+}$и $G_{\mathrm{B}}^{-} Z_{\mathrm{B}}^{-}$в $(4 \mathrm{a})$ сокрашаются и при вычислении $(29)$ используются только соотношения (32), отвечаюшие производным $G_{\mathrm{F}}^{ \pm}$. Итак, с учетом $(32)$ получаем

$$
\begin{aligned}
\langle Q(m)\rangle= & \frac{m}{2 \pi} \int_{-\pi}^{\pi}\left(1+e^{\beta \varepsilon_{q}}\right)^{-1} d q, \\
\left\langle Q^{2}(m)\right\rangle= & \frac{m}{2 \pi} \int_{-\pi}^{\pi}\left(1+e^{\beta \varepsilon_{q}}\right)^{-1} d q+\frac{m^{2}}{(2 \pi)^{2}}\left[\int_{-\pi}^{\pi}\left(1+e^{\beta \varepsilon_{q}}\right)^{-1} d q\right]^{2}- \\
& -\int_{-\pi}^{\pi} \int_{-\pi}^{\pi}\left(Q_{p q}\right)^{2}\left(1+e^{\beta \varepsilon_{p}}\right)^{-1}\left(1+\varepsilon^{\beta \varepsilon_{q}}\right)^{-1} d p d q .
\end{aligned}
$$

Из (33) получаем в термодинамическом пределе

$$
\sigma_{z} \equiv\left\langle\sigma_{z}^{(m)}\right\rangle=1-\frac{1}{\pi} \int_{-\pi}^{\pi} \frac{d q}{1+e^{\beta \varepsilon_{q}}},
$$

где используются определения $\left\langle\sigma_{z}^{(m)}\right\rangle=1-2 \mathcal{D}_{1}\langle Q(m)\rangle$ и $\mathcal{D}_{1} f(m)=f(m)-f(m-1)$. Результат (35) согласуется с намагниченностью $\mathcal{M}_{z}=-\partial F / \partial h$, вычисленной в [4]. Из (34) получаем

$$
\left\langle\sigma_{z}^{(m+1)} \sigma_{z}^{(1)}\right\rangle=\sigma_{z}^{2}-\frac{1}{\pi^{2}}\left|\int_{-\pi}^{\pi} \frac{e^{i m q}}{1+e^{\beta \varepsilon_{q}}} d q\right|^{2}
$$

где используются определения

$$
\begin{gathered}
\left\langle\sigma_{z}^{(m+1)} \sigma_{z}^{(1)}\right\rangle=2 \mathcal{D}_{2}\left\langle Q^{2}(m)\right\rangle+2 \sigma_{z}-1, \\
D_{2} f(m)=f(m+1)-2 f(m)+f(m-1) .
\end{gathered}
$$

Полученные ответы правильно воспроизводят результат [4]. Это свидетельствует в пользу корректности возникающего при вычислении $G(\alpha, m)$ по формулам (4) представления в виде функционального интеграла (13), которое сопровождается граничным условием $(11 \mathrm{~B})$ для $G_{\mathrm{F}}^{ \pm} Z_{\mathrm{F}}^{ \pm}$или аналогичным условием с заменой двух знаков минус на знаки плюс для $G_{\mathrm{B}}^{ \pm} Z_{\mathrm{B}}^{ \pm}$. 


\section{6. ОБСУЖДЕНИЕ}

В работе получено представление для производящей функции статических корреляторов третьих компонент спинов в $X X$-модели Гейзенберга в терминах функциональных интегралов по переменным, подчиняющимся "автоморфному" граничному условию по мнимому времени. Представленная работа примыкает к работе [17], в которой функциональное интегрирование с "автоморфным" граничным условием используется для вычисления индексов некоторых дифференциальных операторов, а также к работе [29], где получены статистические суммы некоторых спиновых моделей на решетке в виде функциональных интегралов по грассмановым полям, преобразуюшимся "автоморфно" при сдвиге мнимого времени на период. Отличие данной работы от [17], [29] состоит в том, что "автоморфное" граничное условие по мнимому времени возникает только для части узлов (нескольких первых) $M$-узельной модели.

Регуляризация гауссовых функциональных интегралов выполнена с использованием обобшенных дзета-функций соответствуюших матричных операторов. При этом удается достичь определенного технического упрошения по сравнению с работой [8], в которой регуляризуемые интегралы домножаются и делятся на вспомогательные конечнократные грассмановы интегралы и дважды сдвигаются переменные интегрирования. Кроме того, показано, что с практической точки зрения (т.е. если требуются только производные по параметру) можно обойти ответ в виде определителя конечномерной матрицы и сразу получить формулу для первой производной производящей функции. Предложенное функциональное представление также делает более прозрачной связь вычисляемых объектов с конечнократным грассмановым интегрированием [9], [10]. В данной работе предложено обобшение технических приемов [17], [29], которое может оказаться полезным в других моделях, где также приходится вычислять вакуумные средние экспонент квадратичных операторов типа $e^{\alpha Q(m)}$.

Благодарности. Автору приятно поблагодарить Н. М. Боголюбова, Ф. Коломо, В. С. Капитонова, В. Е. Корепина, П. П. Кулиша, Ж. М. Майе, Х. Ю. Микеску, А. Г. Пронько, Н. А. Славнова и П. Г. Зографа за интересные обсуждения. Работа частично поддержана грантом РФФИ № 01-01-01045.

\section{Список литературы}

[1] Н. М. Боголюбов, А. Г. Изергин, В. Е. Корепин. Корреляционные функции интегрируемых систем и квантовый метод обратной задачи. М.: Наука, 1992.

[2] A. Lenard. J. Math. Phys. 1964. V. 5. № 7. P. 930-943; 1966. V. 7. № 7. P. 1268-1272.

[3] V. E. Korepin, N. A. Slavnov. Commun. Math. Phys. 1990. V. 129. № 1. P. 103-113.

[4] F. Colomo, A. G. Izergin, V. E. Korepin, V. Tognetti. Phys. Lett. A. 1992. V. 169. № 4. P. 243-247; ТМ $\Phi$. 1993. Т. 94. № 1. С. 19-51.

[5] А. Г. Изергин, А. Р. Итс, В. Е. Корепин, Н. А. Славнов. Алгебра и анализ. 1994. Т. 6. № 2. С. $138-151$.

[6] А. А. Славнов, Л. Д. Фаддеев. Введение в квантовую теорию калибровочных полей. М.: Наука, 1988

[7] В. Н. Попов. Континуальные интегралы в квантовой теории поля и статистической физике. М.: Атомиздат, 1976. 
[8] В. С. Капитонов, К. Н. Ильинский. Зап. научн. семин. ПОМИ. 1995. Т. 224. С. 192-207.

[9] А. Г. Изергин, В. С. Капитонов, Н. А. Китанин. Зап. научн. семин. ПОМИ. 1997. T. 245. C. $173-206$.

[10] В. С. Капитонов, А. Г. Пронько. Зап. научн. семин. ПОМИ. 2000. Т. 269. С. 216-261.

[11] Ф. А. Березин. Метод вторичного квантования. М.: Наука, 1986.

[12] А. Г. Изергин, Н. А. Китанин, Н. А. Славнов. Зап. научн. семин. ПОМИ. 1995. Т. 224. C. $178-191$.

[13] U. Bilstein, B. Wehefritz. J. Phys. A. 1999. V. 32. № 2. P. 191-233; U. Bilstein, V. Rittenberg. J. Phys. A. 2000. V. 33. № 42. P. L381-L385.

[14] V. Korepin, J. Terilla. Quantum Information Processing. 2002. V. 1. P. 225-242; quant-ph/0202054.

[15] N. Kitanine, J. M. Maillet, N. A. Slavnov, V. Terras. Nucl. Phys. B. 2002. V. 642. P. 433-455; hep-th/0203169.

[16] T. Kojima, V. Korepin, N. Slavnov. Commun. Math. Phys. 1997. V. 188. № 3. P. 657-689; V. 189. № 3. P. 709-728; V. E. Korepin, N. A. Slavnov. J. Phys. A. 1998. V. 31. № 46. P. $9283-9295$.

[17] L. Alvarez-Gaumé. Supersymmetry and index theory. In: Supersymmetry. NATO ASI Ser. V. 125. Eds. K. Dietz et. al. New York: Plenum, 1985. P. 1-44.

[18] К. Мальшев. Зап. научн. семин. ПОМИ. 2000. Т. 269. С. 269-291; C. Malyshev. ХХ Heisenberg spin chain and an example of path integral with "automorphic" boundary conditions. hep-th/0204007.

[19] S. W. Hawking. Commun. Math. Phys. 1977. V. 55. № 2. P. 133-148; J. S. Dowker, R. Critchley. Phys. Rev. D. 1976. V. 13. № 12. P. 3224-3232.

[20] C. Хокинг. Интегралы по траекториям в приложении к квантовой гравитации. В сб.: Общая теория относительности. Ред. С. Хокинг, В. Израэль. М.: Мир, 1983. С. 363-406; Б. С. Де Bumm. Квантовая гравитация: новый синтез. В сб.: Общая теория относительности. Ред. С. Хокинг, В. Израэль. М.: Мир, 1983. С. 296-362.

[21] D. B. Ray, I. M. Singer. Adv. Math. 1971. V. 7. № 2. P. 145-210.

[22] E. Corrigan, P. Goddard, H. Osborn, S. Templeton. Nucl. Phys. B. 1979. V. 159. № 3. P. 469-496; H. Osborn. Nucl. Phys. B. 1979. V. 159. № 3. P. 497-511.

[23] A. S. Schwarz. Commun. Math. Phys. 1979. V. 64. № 3. P. 233-268.

[24] B. P. Dolan, Ch. Nash. Commun. Math. Phys. 1992. V. 148. № 1. P. 139-153; R. Kantowski, K. A. Milton. Phys. Rev. D. 1987. V. 36. № 12. P. 3712-3721.

[25] В. Н. Романов, А. С. Швари. ТМФ. 1979. Т. 41. № 2. С. 190-204.

[26] A. С. Швари. Квантовая теория поля и топология. М.: Наука, 1989.

[27] E. Lieb, T. Schultz, D. Mattis. Ann. Phys. 1961. V. 16. № 3. P. 407-466; Th. Niemeijer. Physica. 1967. V. 36. № 3. P. 377-419; 1968. V. 39. № 3. P. 313-326.

[28] W. L. Baily, Jr. Introductory Lectures on Automorphic Forms. Princeton, NJ: Princeton Univ. Press, 1973; И. Р. Шафаревич. Основания алгебраической геометрии. М.: Наука, 1988.

[29] В. Н. Попов, С. А. Федотов. ЖЭТФ. 1988. Т. 94. № 3. С. 183-194.

[30] В. А. Фок. Изв. АН СССР. Сер. физ. 1937. № 4-5. С. 551-568; J. Schwinger. Phys. Rev. 1951. V. 82. № 5. P. 664-679.

[31] W. Magnus, F. Oberhettinger, R. P. Soni. Formulas and Theorems for the Special Functions of Mathematical Physics. Berlin: Springer, 1966.

[32] A. S. Schwarz. Lett. Math. Phys. 1978. V. 2. № 3. P. 247-252.

[33] Ф. Р. Гантмахер. Теория матриц. М.: Наука, 1988.

Поступила в редакцию 23.VIII.2002 г. 